\title{
Scripta
}

\section{La Cancillería de Martín el Humano a través de los Registros Notariorum (1396-1410)}

\section{The Chancery of Martin Human across the Records Notariorum (1396-1410)}

\author{
Ma Milagros Cárcel Ortí / Vicente Pons Alós \\ Vicente.Pons-Alos@uv.es / Milagros.Carcel@uv.es
}

Universitat de València

\begin{abstract}
Resumen: El objetivo de este trabajo es conocer a través de dos registros notariorum del Archivo de la Corona de Aragón quiénes desempeñaron los diferentes cargos de canciller, vicecanciller, regente de cancillería, protonotario, secretario, escribano, archivero, sellador, portero, examinador de notarios, tesorero y otros durante el reinado de Martín I, así como los miembros de su casa y corte como capellán, confesor, consejero, médico y otros, además del personal de otras escribanías reales, señoriales y episcopales.
\end{abstract}

Palabras clave: cancillería real, diplomática real, Corona de Aragón, Martín I, registros de cancillería

\begin{abstract}
The aim of this paper is know across two records notariorum about the Archive of the Crown of Aragon those who recovered the different charges of chancellor, vice chancellor, regent of chancery, prothonotary, secretary, notary, archivist, sealer, doorman, examiner of notaries, treasurer and others during the reign of Martin I, as well as the members of his house and court as chaplain, confessor, counselor, doctor and others, besides the personnel of other royal, lordly and episcopal clerkships.
\end{abstract}

Keywords: Royal chancery, royal diplomatic, Crown of Aragon, Martin I, records of chancery

DATA PRESENTACIÓ: 29/09/2015 ACCEPTACIÓ: 08/12/2015 · PUBLICACIÓ: 26/12/2015

SCRIPTA, Revista internacional de literatura i cultura medieval i moderna, núm. 6 / desembre 2015 / pp. 1 - 23 ISSN: 2340-4841 $\cdot$ doi:10.7203/SCRIPTA.6.7008 
Mª Milagros Cárcel Ortí \& Vicente Pons Alós. La Cancillería de Martín el Humano a través de los Registros Notariorum (1396-1410)

Los estudios sobre la Cancillería de la Corona de Aragón durante el reinado de Martín el Humano (1396-1410) son escasos, a pesar de la abundancia de fuentes: 500 pergaminos, 1700 cartas reales y 248 registros en papel solamente en el Archivo de la Corona de Aragón (Udina Martorell, 1986). Trenchs y Aragó $(1983,16)$ sitúan esta cancillería en la etapa que va desde las Ordinacions de Pedro IV (1344) hasta la muerte de Martín I (1410), caracterizada «por la codificación de los estamentos y funcionarios, por la norma documental y de sellado, por la aparición de tres vicecancilleres con Juan I y por la revitalización de tipos documentales concernientes a la administración en todos sus niveles». Francisco Sevillano Colom en su estudio sobre las cancillerías de Fernando I y Alfonso el Magnánimo analiza también algunas cuestiones relativas al reinado de Martín el Humano y la composición de la misma $(1965,169-216) . M^{a}$. Teresa Ferrer i Mallol ha estudiado también los cargos de su corte y los miembros del Consell Reial (1996, 173-190), aunque sigue siendo el trabajo de Daniel Girona sobre el Itinerario del rey Martín, publicado entre 1911 y 1914, el estudio más completo (1911-1914). El canciller era el cargo más importante del Consejo real y de la escribanía regia con una doble función judicial y administrativa. En 1387 Juan I determinaba que tres vicecancilleres, uno por cada Reino, ayudasen al canciller, normalmente eran doctores en leyes de prestigio. Los regentes de cancillería, por su parte, sustituían eventualmente al canciller o vicecancilleres y también solían ser juristas. El protonotario era el jefe del personal de la escribanía regia, velando por la buena redacción de los documentos, la recaudación del derecho de sello y llevaba el Dietario con los desplazamientos del monarca, contaba además con la ayuda de un lugarteniente y dos secretarios, que suscribían las cláusulas de mandato. Finalmente, el archivero, «tenenti claves archivi civitatis Barchinone», uno de los cargos más antiguos, era uno de los escribanos de mandato encargado de tener las llaves del archivo y de los cofres donde se custodiaban los documentos y registros. Debía encargarse de poner títulos a los registros, numerar los folios, redactar índices y la conservación y reparación de los registros, controlando que no se sacasen copias sin la debida autorización.

Un primer cargo de sellador de la escribanía fue ampliado a dos selladores ordinarios y dos extraordinarios, ayudados por el receptor de los derechos del sello, encargado de asegurar el pago de dicho derecho, y por el scalfatore cere o calefactor cere scribanie domini regis.

Los registros de notariorum de Martín el Humano documentan también a los peticionarii, que aunque Sevillano Colom indica que comienzan con Alfonso V, ya aparecen con anterioridad. Finalmente, los virgarium audiencie domini regis y porteros, que a veces actuaban como alguaciles.

Con Martín I en 1409 se reduce el número de escribanos de mandato a 12, aunque más adelante dispuso que estos 12 fueran ordinarios, y un número que osciló entre 6 y 12 extraordinarios o suplentes de los primeros. Estos últimos, tras 4 años de aprendizaje entraban como extraordinarios y al producirse una vacante pasaban a ordinarios. Lo mismo ocurría con los escribanos de registro, 8 ordinarios y 8 extraordinarios. No es fácil distinguir unos de otros, aunque tanto los de mandato como los de registro son normalmente notarios. Sólo en dos casos los registros de Notariorum

SCRIPTA, Revista internacional de literatura i cultura medieval i moderna, núm. 6 / desembre 2015 / pp. 1 - 23 ISSN: 2340-4841 · doi:10.7203/SCRIPTA.6.7008 
Mª Milagros Cárcel Ortí \& Vicente Pons Alós. La Cancillería de Martín el Humano a través de los Registros Notariorum (1396-1410)

especifican que ambos escribanos son de mandato. La fórmula mandavit mibi identificaba a los secretarios, mientras que las de mandato regio o mandato domini regis remitía a los escribanos, de mandato o de registro. La cantidad de escribanos de registro documentados se justifica porque los cualificados y laboriosos perduraban, mientras que los menos habilidosos cesaban con rapidez. Estos eran amanuenses a las órdenes de los de mandato, como indica el nombre.

La coincidencia con el movimiento prerrenacentista y la influencia de la corte pontificia de Aviñón dará como resultado mayores exigencias estéticas y literarias que se reflejan en los documentos (Conde y Delgado de Molina \& Cárcel Ortí, 1996, 284).

Entre los registros de Cancillería de notariorum conservados en el Archivo de la Corona de Aragón destacan dos para el periodo de Martín el Humano (Pons Alós, 2015). ${ }^{1}$ Comprenden los nombramientos de notarios reales de toda la Corona de Aragón y, a partir de su reinado, éstos se desdoblarán en dos, uno asentando la expedición de las cartas de los nombramientos reales (pro cartis), otro haciendo constancia del juramento (pro iuramentis), razón por la cual este segundo empieza con la relación de los ocho puntos quod iurent notarii noviter creati infrascripta tenere et observare, cartas que debían presentar al justicia civil y juramentos que, en muchos casos, se hacían ya ante el justicia local correspondiente (Torra Pérez, 1994, 179-194). No todos los notarios a los que se les concede la carta de nombramiento realizan después el juramento en la propia cancillería. Ambos registros permiten conocer el itinerario y características del proceso de nombramiento de notarios reales con Martín el Humano, a la vez que establecer su prosopografía. Si el primer registro de Notariorum, entre 1351 y 1368, separa los nombramientos de los notarios de Aragón, Valencia y Cataluña, los siguientes siguen el orden cronológico mezclando los nombramientos de todo el ámbito de la Corona de Aragón, incluyendo el sur de Francia y Cerdeña (Pons Alós, 2012, 31-86).

El registro pro cartis presenta una estructura simple, a modo de acta breve del nombramiento, con indicación de la fecha tópica y crónica, el nombre del notario, el lugar de procedencia, tipo y ámbito de su nombramiento, la constatación de la firma y aceptación de obligaciones: firmarum et obligationum notariorum, y el nombre, condición y origen del fideiusor que se presenta como aval. A veces sobre el nombre del notario o del fideiusor se coloca una pequeña rúbrica, que siguiendo la práctica notarial, parece indicar su presencia. Otras, en muy pocos casos, la abreviatura iur(avit), señalando que juraron el cargo. En los márgenes, excepcionalmente, se indica el pago de la tasa: solvit, generalmente 2 sueldos, la exención: non solvit, y en algún caso la nota marginal indica la

\footnotetext{
* Agradecemos al amigo y profesor de Historia Medieval Dr. Mateu Rodrigo Lizondo la ayuda y correcciones en la realización de este trabajo. Este trabajo ha sido realizado en el marco del proyecto de investigación "Documentación real de la Edad Media conservada en archivos valencianos: edición crítica y estudio” del Ministerio de Economía y Competitividad. Ref. HAR2012-36656.

1 ACA. Real Cancillería 2304, Notariorum. Pro iuramentis (144 fol.) y 2305, Notariorum. Pro cartis (201 fol.). La diferencia de extensión se debe a que no todos los notarios a los que se les concede la carta de nombramiento realizan después el juramento en la propia Cancillería.
} 
Mª Milagros Cárcel Ortí \& Vicente Pons Alós. La Cancillería de Martín el Humano a través de los Registros Notariorum (1396-1410)

nulidad del asiento: non venit ad effectum. Ambos registros, entre 1397 y 1410, no siempre están ordenados cronológicamente y no solamente suponen una fuente interesante para reconstruir el itinerario real (Girona, 1910-1914), sino también ofrecen una información exhaustiva sobre la composición de la cancillería real, cuyos personajes firman generalmente como testigos o como fideiusores, así como sobre el proceso de gestación de la documentación, que aparece ampliamente manifiesto en el registro «pro cartis».

Los inicios de la cancillería de Martín el Humano como rey fueron conflictivos como lo demuestra el proceso llevado a cabo contra algunos de sus miembros, aunque la mayoría de ellos fueran después perdonados y regresaran a sus puestos (Mitjà, 1957-58, 375-417). Previamente, como príncipe, duque de Montalbà, conde de Luna y señor de Segorbe, el infante tuvo su propia escribanía. Así en 1391 se documenta a Berenguer de Cruilles y Bernat de Cabrera, como consejeros y camarlengos suyos, a Berenguer Sarta, como notario público y su protonotario, o al mismo Sarta, Ramon Sescomes y Joan de Bossegays, como sus escribanos (Trenchs \& Aragó, 1983).

Según Ma . T. Ferrer la llegada al trono de Martín el Humano fue aprovechada por las ciudades de Barcelona y Valencia, que le habían dado una ayuda fundamental para que sus derechos sucesorios fuesen respetados, para imponer una mayor presencia de estas dos ciudades en los órganos de poder decisivos: cancillería y tesorería (Ferrer i Mallol, 1996, 180). Efectivamente, en el listado de miembros de la cancillería aparece un número considerable de personajes oriundos de estas ciudades, especialmente la primera (Günzburg Moll, 2001, 47-55). Además, continúa destacando una notable endogamia: los hermanos Joan y Nicolau Esquerdo, ambos escribanos de la escribanía y el segundo también scalfatore cere, los también hermanos Pere Sescomes, escribano de cancillería, y el protonotario Ramon Sescomes, Bernat y Bartomeu Sirvent, también protonotario, el escribano Bertrán de Tudela y Joan de Tudela, escribano y secretario real, Jaume y Joan Bossegays, Francesc y Pere Pellisser, el también escribano Pere Roma, pariente del vicecanciller Francesc Roma, el archivero Gabriel Segarra, hijo de Berenguer, o Pere Desplá y su padre homónimo, ambos selladores y calentadores de la cera. No es mera casualidad la repetición de apellidos.

Otra característica importante de la cancillería de Martín el Humano, como consecuencia de la mayor complejidad administrativa, es el aumento del personal así como su diversificación. De uno a tres vicecancilleres de los reinados anteriores, que no siempre actuaban a la vez, se pasará a cuatro: Macià Castelló, vicecanciller primero, quien sustituía a menudo al canciller, ausente de la corte, especialmente con Eñego de Vallterra; Domingo Mascó, vicecanciller para Valencia, Ramon de França, para Aragón, y Esperandeu de Cardona, para Cataluña. Además de los vicecancilleres, había un buen número de regentes de la cancillería (6) que ayudaban y sustituían al canciller y a éstos en sus ausencias (Ferrer i Mallol, 1996, 179). El número de escribanos, que se había visto incrementado ya con Pedro IV, prácticamente se triplica, así como la pluralidad de oficios: escribanos que a la vez son de domo, como Capdevila, o que ocupan el cargo también de selladores en determinados momentos. 
Ma Milagros Cárcel Ortí \& Vicente Pons Alós. La Cancillería de Martín el Humano a través de los Registros Notariorum (1396-1410)

Los registros notariorum de Martín el Humano (1356-1410) documentan también el personal de otras escribanías reales, señoriales y episcopales. De la del infante Martín el Joven (1376-1409), rey de Sicilia, del que se citan cuatro escribanos: Antoni Bruch, Ramon Marí, Jaume Menor y Pere Companyó. De la reina Violant de Bar (+1431), viuda de Juan I y hermana de Carlos V de Francia, de la que conocemos los nombres de sus protonotarios, tesorero, cambrers, escribanos, parte del personal de su casa y de su confesor, el franciscano Joan Eiximenis. De la reina María de Luna (+1406), primera esposa de Martín el Humano, su camarlengo, tesorero, y algunos de sus escribanos. De la infanta Leonor, hermana del rey Martín, su despensero. Finalmente, también de la escribanía de Isabel de Foix, esposa de Arquimbald de Grailly, conde de Benanges, y heredera del condado de Foix y Bearn, tras la muerte sin hijos de su hermano Mateu I de Foix en 1398, los registros remiten a uno de los escuderos de su mensa; y de la escribanía episcopal Valentina al secretario y proveedor de la casa del obispo.

\section{A. Cancillería}

\section{CANCILLER}

- Fernández de Heredia, García (1387-1399), canónigo de Mallorca, obispo de Vich (13771383) y arzobispo de Zaragoza (1383-1411) y sobrino de Juan Fernández de Heredia. Canciller también de Juan I, fue asesinado al tomar partido por Luis de Anjou.

- Valiterra, Eñego de, obispo de Girona (1362-1369), de Segorbe (1369-1380) y arzobispo de Tarragona (1380-1407). Ya fue canciller del infante Martín (1400-1407), según Ma Teresa Ferrer, de manera más nominal que real.

- Blanes, Francesc de (Blanis), Franciscus, cancellarius (1401, jun.-1410, ene. 9), doctor en decretos y hermano de Vidal de Blanes, referendari de Benedicto XIII, fue consejero y promovedor del rey al menos desde 1399. Fue además embajador en Aviñón y obispo de Girona (1408) y Barcelona (1408-+1410) sucesivamente.

\section{VICECANCILLER}

- Valiseca, Guillem de (1393-...), caballero de Barcelona.

- CARdona, Esperandeu (1389-1396/1402-1410), vicecanciller por Cataluña

- Castelló, Macià, Mathias Castilionis, vicecancellarius; Matias, vicecancellarius (1397-1403), vicecanciller primero, sustituía a menudo al canciller ausente, especialmente en la etapa de Eñego de Vallterra.

- FRANÇA, Ramon de (1386-...), vicecanciller por Aragón.

- MAscó, Domingo (1387-+1427), jurista, nombrado vicecanceller por Valencia. ${ }^{2}$

- Miquel, Bernat (1396-1397...1406). ${ }^{3}$

2 Había sido ya vicecanciller con Juan I y es también consejero del rey Martín (ACA. Real Cancillería, 1920, fol. 85) (Graullera Sanz, 2000).

3 Parece que también Pere Serra, cardenal de Catania (1397-+1405), doctor en derecho, acompañó a Martín I a Sicilia actuando como vicecanciller (1392). Fue uno de los principales consejeros que el infante Martín dejó a su hijo a su vuelta a Cataluña (Pons Alós, 2005). 
Ma Milagros Cárcel Ortí \& Vicente Pons Alós. La Cancillería de Martín el Humano a través de los Registros Notariorum (1396-1410)

\section{Regente de CANCILleria}

- Miquel, Bernat (1397, nov. 6-1409, feb. 27), in legibus licenciatus, consiliarius et promotor negociorum curie dicti domini regis ipsiusque cancellariam regens

- Canyelles, Nicolau de (1400, feb. 26-1404, sep. 25), Nicolaus de Canellis/Nicholaus de Canyellis, regens Cancellarie; Nicolaus, promotor ${ }^{4}$

- Ximénez de Galloç, Fernando (1400, may. 30-1402, feb. 1).

- SAnt Dionís, Dalmau (1400/1403, sep. 26-1407, dic. 4), legum doctor curie dicti domini regis, auditor ac cancellariam eiusdem domini nunc regens; Dalmacius vidit. ${ }^{5}$

- Coll, Pere de (Petrus de Colle), auditor ut regentem cancellarie (1402, abr. 13).

- Saranyana, Joan (1404, mar. 19), regens cancellarie.

- CARdona, Esperandeu (1404, may. 23-1410, may. 21).

- Pere, Bonanat (1404, oct. 12-1410, may. 20), Bonanatus Petri, regens cancellarie.

- Olzina, Pere (1405, dic. 22-1407, oct. 29), Vidit Petrus Olžina .

\section{Protonotario o guardasellos}

- SiRvent, Bartomeu (1390-1401).

- Besanta, Pere (1403).

-Sescomes, Ramón (Ces Comes/Sacomal de Cumbis, Raimundus), (...1406-1410), notarius auctoritate regia per totam terram et dominationem. Continuó ocupando este cargo con Fernando I, que le confirmó en el mismo el 9 de ago. de 1412. Lo será luego también de la reina María, esposa de Alfonso el Magnánimo.

\section{Secretarios del Rey (SECRETARII DOMINI REGIS)}

- SARTA, Berenguer de (Berengarius) (v) (1397-1404, jul. 19) (t-f). ${ }^{6}$

- Tudela, Joan de (Johannes de) (1397-1410/1403, ene. 20-1409, dic. 9)(t-f). ${ }^{7}$

- Ponç, Guillem (Guillermus Poncii) (1402.../1397-1400). ${ }^{8}$

- SAFont, Antoni de (Anthonius de Fontis) (1397-1406), escribano real y de domo.9

- Metge, Bernat (Medici, Bernardus) (1408, diciembre 27-1410, febrero 27).

6. LUGARTENIENTES DE PROTONOTARIO (LOCUMTENENTES PROTHONOTARII)

- TAvascà, Jaume (Jacobus Tavaschani, Tavescan) (1406, ago. 12-1408, jul. 5-1410) (t.).

- Bellvehí, Guillem de (Bellvehi).

4 Era doctor en leyes. Algunos autores se refieren a él como Narcís de Canyelles, e incluso Arnau de Canyelles (Trenchs \& Aragó, 1983, 54).

5 In domo habitaciones rectoris Gandie que est iuxta et satis prope ecclesiam Sancti Johannis dicte civitatis in qua hospitabatur. Doctor, consejero, auditor y regente de la cancillería, en algunos momentos desempeña las funciones del canciller (1400).

6 Era protonotario del rey Martín I cuando todavía era duque de Montblanc (1391). Petrus Sarta, hijo suyo, es escribano de la escribanía regia.

7 Petrus Torrent, cum dicto Johannem comorans (1409). Fue también secretario de Alfonso el Magnánimo (Sevillano Colom, 1965, 196-197, 205).

8 Notarius publicum per totam terram et dominacionem, había sido escribano de mandamiento de Pedro IV (1380-1386) y escribano secretario de Juan I (1391-1396) (Rubió i Balaguer, 1961, 72; D’Arienzo, 1974, 194).

9 Ya era escribano real con Juan I (1388-1396) y siguió siendo escribano de mandamiento con Fernando I y Alfonso el Magnánimo (1416-1418). 
Ma Milagros Cárcel Ortí \& Vicente Pons Alós. La Cancillería de Martín el Humano a través de los Registros Notariorum (1396-1410)

7. Escribanos (DE SCRIBANIA (DICTI) DOMINI REGIS/DE SCRIBANLA REGIA/ SCRIPTORES SCRIBANIE) ${ }^{10}$

- Alemany, Joan (1399, jun. 19) (t.).

- AlçAmora, Mateu d' (1408-1410) (e.m.).

- Angusoles, Martí d' (d'Ang(l)usolis, Angulosis), not. (1398, oct. 22-1409, nov. 6) (t.). ${ }^{11}$

- Asp, Joan d' (1402, abr. 15) (t.).

- Assió, Joan d' (Assins, Assió, Dasiu, Dassió) (1399, may. 14- 1409, nov. 8) (t.); (1410) (s.e.r.).

- Ansó, Blasco de (Danson, d'Ansó, Averso?), not. (1400, oct. 30-1408, nov. 24) (t.); ${ }^{12}$ escribano auditor (1408-1410).

- BARÓ, Ramon (Baromi), not. (1397, nov. 17-1409, oct. 3) (t.); (1410) (s.e.m.).

- BAs, Ramon, not. (1397, sept. 13-1402, mar. 9) (t.) ; (1410) (e.r.).

- Besiers, Bartomeu (Beses, Basers), not. (1397, sept. 13-1406, ago. 21) (t-f).

- BLANCH, Berenguer (1397, jun. 22) (t.).

- Bossegays, Jaume (Jacobus de Bossagay/Bossaganys), not. (1397, dic. 6- 1410, may. 31) (t.).

- Bossegays, Joan de (1397, abr. 26-1400, jun. 5) (t-f). ${ }^{13}$

- Busquets, Berenguer de (1398, febrero 26-1401, junio 3).

- Calonge, Francesc (1410, ene. 28) (t.).

- Canet, Bernat de (de Caneto), not. (1397, nov. 17- 1408, dic. 19) (t.).

- Capdevila, Pere de, not. (1397, dic. 11- 1408, ene. 25) (t.).

- Cardona, Gabriel de (1406).

- CAyllet, Lluís (1408, nov. 19) (f.).

- Comes, Pere (Comes) (1407, jun. 23-dic. 16) (t.).

- Creixell, Joan (de Crexello), not. (1397, sept. 14-1406, sept. 2) (t.); (-1410) (e.r.).

- Cudina, Salvador (1406, mar. 20) (t.).

- Desmas, Pau (Dezmas) (-1410, ene. 15) (t.).

- Dalmau, Joan (Dalmacii) (-1410, may. 23) (f.). ${ }^{14}$

- Desnoguers, Pere (Deznoguer(t.)s, Noguer), not. (1406, ene. 27-1408, febr. 8) (t.) ; (1410) (s.e.r.).

- Desplà, Pere (Deaplà) (1400, may. 15- 1406, may. 24) (t.).

- Despuig, Nicolau (de Podio) (1400, oct. 30) (t.).

- Despuig, Salvador (de Podio) (1404, jul. 14).

- Despujol, Joan (dez Puiol), not. (1405, nov. 18-1409, may. 13) (t-f).

- DíAz, Diego (1409, ago. 3) (t.).

- Esquerdo, Joan, not. (1397, dic. 6-1407, may. 20) (t-f); ${ }^{15}$ (1410) (e.r.)

10 Discreti.

11 Hay un notario de Zaragoza con el mismo nombre (1403, sept. 11) (f.).

12 Comorans cum honorabili Jacobo Tavascani.

13 Ya era escribano del infante Martí (1388-1390).

14 Oriundo de Gerona, es nombrado notario per totam terram et dominacionem domini regis en la fecha dada.

15 Hermano de Nicolás. Proceden de Valencia.

SCRIPTA, Revista internacional de literatura i cultura medieval i moderna, núm. 6 / desembre 2015 / pp. 1 - 23 
Mª Milagros Cárcel Ortí \& Vicente Pons Alós. La Cancillería de Martín el Humano a través de los Registros Notariorum (1396-1410)

- Esquerdo, Nicolau (Squert/Squerdo/Exquierdo), not. (1399, sept. 9-1409, nov. 21) (t.). ${ }^{16}$

- EXERICH, Domingo (1399, febr. 13-1402, jun. 29) (t-f).

- Ferrandell, Mateus (Ferrandelli) (1405, nov. 18-1408, ago. 6) (f.).

- GARCIA, Diego (1398, may. 30-1410, mar. 20) (t.), ${ }^{17}$ (1410) (e.r.); De 1415 a 1436 fue archivero.

- Gaver, Ramon de (de Gavero), (1399, may. 14- 1410, abr. 19) (t-f).

- GIL, Joan Miquel (Johannes Michael Egidii), not. (1397, nov. 6- 1410, abr. 4) (t.).

- Girgós, Tomàs (Thomasius Girgorsii/ Gircós), (1397 sept. 5-1400, febr. 9) (t.).

- Gostemps, Berenguer de (1400, dic. 9-1410, mar. 22) (t-f); ${ }^{18}$ (1410) (e.m.).

- GrÀcia, Bartomeu de, not. (1397, sept. 29-1408, dic. 5) (t.).

- Gras, Bartomeu (1397, septiembre 26-1409, jul. 15).

- Gallart, Martí (G(u)allardi, Gallart) (1398, ene. 21-1407, may. 20) (t-f). ${ }^{19}$

- Guardiola, Francesc de (Gordiola/ de Guardiolis/ Guardiolis) (1397, nov. 6-1402, jun. 14) (t.).

- GuAYte(R)s, Ramon (Guayceres), not. (1400, ene. 17-1410, may. 23) (t.); (1410) (s.e.r.)

- Guitart, Guillem (Guitardi, Guillermus, scriptor) (1403, may. 12-1409, diciembre 17) (t.); (1410) (s.e.m.).

-Just, Maties (Just), scriptor (1402, febr. 1- 1409, jun. 13) (t.); (1410) (s.e.m.).

- MAÇA, Miquel de (Maçes/Maça), not. (1397, jun. 23-1402, may. 13) (t.). ${ }^{20}$

- Marín, Pere (1400, abr. 2) (t.).

- Mercader, Francesc, not. (1403, abr. 23) (t.).

- Metge, Bernat (Medici, Bernardus) (1407, may. 2- 1408, ene. 4).

- Montreal, Guillem de (Montreal/Muntreal) (1401, nov. 22- 1410, may. 23) (t.); (1410) (s.e.r.).

- Montsó, Mateu (1398, ene. 14) (t.).

- Manyosa, Arnau (Manyosa) (1399, febr. 24-mar. 5) (t.).

- Nicolas, Pau de (Nicholas), not. (1405, abr. 24-1409, dic. 17) (t-f). ${ }^{21}$

- Olivan, Garcia de (-1410, mar. 22) (t.).

- Olzinelis, Rafael de (Ulizinellis) (1404, sept. 6-1408-may. 21) (t.).

- Oros, Miquel d' (Aros, Doros, Doroz) (1406, abr. 1- 1410, mar. 12) (t-f).

- Ortigues, Huguet d' (Huguetus de Urtigi(i)s), not. (1397, sep. 5- 1409, ago. 9) (t.). ${ }^{22}$

- Pellisser, Francesc, scriptor Barchinone ${ }^{23}$ (1399, may. 17-1410, febr. 17) (t.).

- Pellisser, Pere (1410, ene. 15) (t.).

16 Hermano de Joan.

17 Es nombrado notario per totam terram et dominacionem domini regis el 15 de ago. de 1405.

18 Filius Berengarii de Gostemps. Padre e hijo coinciden como t. y f. el 14 de abr. de 1404.

19 En 10 de jul. de 1400 aparece como scriptor mandati.

20 Notarius noviter creatus per totam terram et dominacionem domini regis Aragonum.

21 Canellas \& Trenchs, 1988, 75; Rubió i Balaguer, 1964, 20. Persona de confianza de Fernando I, pasará a ser secretario interino en el proceso entre varios vecinos de Zaragoza, Calatayud, Teruel y Daroca, por el asesinato del arzobispo de Zaragoza con motivo de los bandos en el interregno, lo que le valió en 1412 una recompensa del rey de 100 florines de oro. Secretario interino también en el proceso contra el conde de Urgell. Estuvo con el monarca hasta su muerte el 2 de abril de 1416, recibió su última voluntad y continuó siendo secretario de Alfonso V hasta 1419.

22 Autor de unas Consilia iuris (Canellas \& Trenchs, 1988, 61).

2314 febr. 1408: scriptor comorans cum domino protonotario.

SCRIPTA, Revista internacional de literatura i cultura medieval i moderna, núm. 6 / desembre 2015 / pp. 1 - 23 ISSN: 2340-4841 · doi:10.7203/SCRIPTA.6.7008 
Ma Milagros Cárcel Ortí \& Vicente Pons Alós. La Cancillería de Martín el Humano a través de los Registros Notariorum (1396-1410)

- Peralta, Pasqual de, not. (1401, abr. 16-1410, ene. 16) (t.). ${ }^{24}$

- PÉres, Miquel (Petri) (1401, jul. 8-1404, jul. 26) (t.).

- Péres, Ramon, not. (1397, jun. 8-1398, mar. 14) (t.); (1410) (e.r.).

- PONÇ, Guillem (Guillermus Pontii) (1398, marzo15-1405, marzo 24).

- Despont, Pere (Petrus de Ponte) (1398, abr. 8/1408, febr. 15-1410, may. 31) (t.); (1410) (e.m.).

- Puig-Grós, Simeó de (Podiogrosso), not. (1401, abr. 14- 1410, febr. 25) (t.). ${ }^{25}$

- Pujol, Pere (1406, may. 24-1407, jun. 13) (t.).

- PuYo, Nicholás de, not. (1402, febr. 3) (f.).

- Rigau, Antonis, not. (1401, may. 29-1402, febr. 3) (t-f).

- Riglos, Juan de (1402, abr. 13); (-1410) (s.e.r.).

- Roma, Pere, not. (1397, dic. 17-1410, abr. 13) (t.).

- Rosset, Pere (Rosseti), scriptor (1399, febr. 13-1410, ene. 16) (t.).

- Roure, Pere (1407, ene. 24) (t.).

- SACOMA, Pere (de Cumba) (1407, jul. 13) (t.).

- SALA, Jaume, not. (1397, jun. 22-1410, abr. 4) (t.). ${ }^{26}$

- SANCHIS, Domingo, alias Solà (1399, ago. 23) (t.).

- Saplana, Bernat (1398, febr. 20 -1409, dic. 9) (t.); (1410) (s.e.m.).

- Sarrablo, Pedro (Serraulo, Serralbo) (1398, jun. 10- 1401, jul. 15) (t.).

- SARTA, Berenguer (1398, marzo 26). ${ }^{27}$

- SARTA, Pere (1402, nov. 19- 1405, nov. 4) (t.). ${ }^{28}$

- SAvila, Salvador (Vila), not. (1404, ago. 16- 1410 mar. 10) (t.).; ${ }^{29}(1410)$ (s.e.r.).

- Sescomes, Ramon (Ces Comes/Sacoma/ de Cumbis, Raimundus), (1396, junio 24- 1405, oct. 3), notarius auctoritate regia per totam terram et dominationem. Más tarde sellador de la Cancillería. ${ }^{30}$

- Sió, Pere de (1403, abr. 7) (t.).

- SiRvent, Bernat (1397, dic. 17-1399, may. 19) (t.).

- Sisó, Joan de (1404, ene. 19) (t.).

- Subirats, Pere (1398, mar. 12-1410, ene. 16) (t.) ; (1410) (e.m.).

- Solivelda, Francesc de (Olivera) (1399, abr. 17- 1404, ago. 8) (t.).

- Sorita, Antoni (1408, may. 17-sept. 18) (f.).

- TALLET, Lluís, not. (1407, dic. 29-31) (t.).

- TARBA, Martín de, not. (1399, febr. 21-1400, abr. 13) (t-f).

- TavascÀ, Jaume (Jacobus Tavaschani/Tavescan) (1406, ago. 12-1408, jul. 5) (t.).

- Tomàs, Pere (1408-1410) (e.m.).

24 El 5 de febr. de 1401 aparece como oriundo de Barcelona y es nombrado notario per totam terram et dominationem domini regis. El 1 de ago. de 1402 aparece otro notario de Valencia del mismo nombre, que podría ser su padre.

25 El 11 de ene. de 1401, oriundo de Barcelona, es creado notarius per totam terram et dominacionem domini regis, y el 14 de abr. del mismo año aparece como comorans cum Francesc Fonolleda, scriptor domini regis.

26 Oriundo de Valencia.

27 Entre 1383 y 1391 se le documenta ya como escribano del infante Martín.

28 Era hijo de Berenguer Sarta, secretario del rey.

29 Oriundo de Barcelona, es nombrada notario per totam terram et dominationem domini regis el 2 de novembre de 1405 . El 5 de mar. de 1408 aparece también un Salvador Çavila, civem Barchinone (f.).

30 Entre 1383 y 1392 ya se le documenta como escribano del infante Martín.

SCRIPTA, Revista internacional de literatura i cultura medieval i moderna, núm. 6 / desembre 2015 / pp. 1 - 23 
Mª Milagros Cárcel Ortí \& Vicente Pons Alós. La Cancillería de Martín el Humano a través de los Registros Notariorum (1396-1410)

- Torrelles, Pere de (1409, ene. 20)

- Tudela, Bertran (Todela), not. (1397, dic. 20- 1401, oct. 31) (t.).

-Tudela, Joan de (1401, diciembre 8- 1408, noviembre 7). Secretario de Alfonso V $(\ldots 1426 \ldots)$.

- VAlLfogó, Mateu (1408-1410) (e.m.).

- Vilagut, Joan de, not. (1398, oct. 20-1410, may. 23) (t.).

- Vilella, Joan (Villella), (1399, ago. 1- 1408, abr. 13) (t.); (1410) (s.e.m.).

- Vilarreial, Guillem de (1405, nov. 16) (t.).

\section{SCRIPTORES DOMINI REGIS}

- Arcos, Pere d' (Arco), not. (1397, nov. 29-1402, sept. 7) (t.); (-1410) (e.m.).

- Arinyo, Francesc d' (1406, nov. 29-1407, oct. 31) (t.). ${ }^{31}$ Secretario de Alfonso V desde 1418 hasta 1429 (+29-XII), le acompañó en sus campañas en Cerdeña y en Italia. ${ }^{32}$

- Aznar de Ansó, Blasco (Azৃar), not. (1401, febr. 20-1408, abr. 10) (t.).

- Balaguer de Picanyes, Joan (1405, nov. 16) (f.).

- Besiers, Bartomeu de (de Beses), not. (1397, sept. 22- 1399, may. 31) (t.).

- Besanta, Pere de (1398, jul. 29) (t.)

- Capdevila, Pere de (Capiteville) (1405, nov. 16) (t.).

- Casanova, Romeu de (1397, sept. 22-1400, dic. 18) (t.).

- Castell, Narcís (1398, ago. 22-1400, ago. 2) (f.).

- Climent, Domingo (1397, jun. 23-1408, nov. 12) (f.).

- Company, Bernat (1402, abr. 15) (f.).

- Coscó, Antoni, not. (1401, febr. 16) (f.).

- Despou, Pere (de Puteo) (1397, dic. 16) (t.).

- Espigoler, Berenguer (Spigoler), (1406, abr. 5-1407, oct. 1) (t-f). ${ }^{33}$ Fue secretario después.

- Exulbe, Lluís de (1405, nov. 16-1408, abr. 13) (t.) ; (1410) (s.e.m.).

- Fonolleda, Francesc de (1401, abr. 14) (t.); ${ }^{34}$ (1410) (s.e.m.)

- Freixenet, Bernat de (Fraxanet) (1403, jun. 26-1409, ene. 20) (t-f) ; (1410) (s.e.m.).

- GaLlart, Martí (1402, ene. 28-29) (t-f); (1410) (s.e.m.).

- Gibel·Lí, Guillem (1408, abr. 12) (t.).

- Jaume, Pere (1405, mar. 23) (f.).

- Leytago, Joan (Laytago/Litago) (1402, febr. 3-1407, jul. 13) (t.); ${ }^{35}$ (1410) (e.m.).

- Manyosa, Arnau de (Mayasa) (1397, nov. 9-1405, nov. 18) (t.); (1410) (e.m.).

31 Canellas \& Trenchs, 1988, 97-98.

32 Speranza, 2015, 435.

33 El 17 de ene. de 1402 era notario de Barcelona.

3414 de abr. de 1401 aparece como comorans cum Simeón de Podiogrosso, de scribania regia.

35 El 5 de ago. de 1404 Johannes Mathei et Dominicus Climenti, scriptores, comorans cum discreto Johannem de Leytago (t.). Fue también escribano de Alfonso de Aragón, conde de Denia y lloctinent reial d'A ragó. 
Mª Milagros Cárcel Ortí \& Vicente Pons Alós. La Cancillería de Martín el Humano a través de los Registros Notariorum (1396-1410)

- Miragall, Pere (Miragall), not. (1399, ene. 20- 1408, jun. 8) (t-f); ${ }^{36}$ (1410) (e.m.). Fue secretario después.

- Mascaró, Gabriel (Mascharon/Masqueroni), not. (1402, jun. 26-1408, may. 13) (f.); (1410) (e.m.).

- Montoliu, Berenguer de (1407, nov. 4) (f.).

- Ortiguer, Pere (1400, jul. 16) (f.).

- Ortigues, Huguet (Ortigiis) (1408-1410) (e.r.).

- PÉres, Miquel (1407, dic. 4) (t.).

- Postigo, Joan de (1400, may. 9) (t.).

- PrunYó, Guillem (1408-1410) (e.r.).

- ReAL, Pere (1401, may. 29- jun. 6) (t.).

- Ros, Bernat (1401, febr. 16) (t.).

-SAFont, Anthoni (Anthonius de Fonte/Fontis) (1399, abr. 27-1407, marzo 21) (t-f) ; (1410) (e.m.).

- SANTES, Jaume de, (v) (1397, ago. 4-dic. 23) (t.).

- SANCHEZ, Eiximen (1398, nov. 4-1400, abr. 9) (t-f).

- SARTA, Pere (1402, ene. 5-29) (t.). ${ }^{37}$

- Tresserres, Berenguer de (Trasserris), (v) (1399, abr. 10-1400, may. 9) (t.); 1410 (s.e.m.)

\section{ARCHIVERO}

- Segarra, Gabriel (1392-1410) (tenens claves archivii civitatis Barchinone), hijo de Berenguer Segarra y copista del monarca. Lo seguirá siendo hasta 1415.

- Relat, Gabriel de, not. (tinent les scriptures) (1409, mar. 26) (t.)

10. Otros cargos

10.1. Peticionarios/PeticionariI ${ }^{38}$

- Capdevila, Pere (1404, sept. 27) (t.).

- Girgós, Tomàs (Girgosii) (1398, mar. 28-1399, dic. 20) (t.).

10.2. SELLADORES/SIGILLATORES SCRIBANIE DOMINI REGIS ${ }^{39}$

- BAsset, Bartomeu, not. (1398, sept. 30) (t.).

- Canet, Bernat (1397, sept. 13- 1409, febr. 18 ; 1410) (t-f)..$^{40}$

- Sescomes, Pere de (Cumbis/Petrus de/Comes/Ces Comes), (1407, ago. 24-1410, febr. 4) (t.).

36 Scriptor mandati. Ya era escribano de Juan I (1385-1391) y se le documenta como tal hasta 1416.

37 Hijo de Berenguer Sarta, secretarius domini regis.

38 Eran los encargados de recibir las súplicas en la cancillería y algunas veces alternaban su función con la de escribanos (Trenchs \& Aragó, 1983, 75).

39 Discreti.

40 Comorans cum Bernardo: Marchus Pellicer.

SCRIPTA, Revista internacional de literatura i cultura medieval i moderna, núm. 6 / desembre 2015 / pp. 1 - 23 
Ma Milagros Cárcel Ortí \& Vicente Pons Alós. La Cancillería de Martín el Humano a través de los Registros Notariorum (1396-1410)

- GIL, Miquel (Egidii/Gili), (1397, sept. 14- 1410, may. 30) (t.).

- GrÀCIA, Bartomeu de, not. (1398, jun. 9-1409, jul. 20; 1410)) (t.).

- Desplà, Pere (Petrus de Plano) (1397-1401, ene. 19) (t.).

- Guardiola, Francesc de (1408-1410).

10.3. CALENTADOR DE LA CERA/SCALFATOR CERE/ CALEFACTOR CERE SCRIBANIE DOMINI REGIS)

- Esquerdo, Nicolau (1399, abr. 8-1405, dic. 4; 1410) (t.).

10.4. CORREOS / CURSORES SCRIBANIE REGIS/REGIE

- ARNAU, Guillem, cursor domini regis (1398, ene. 3) (t.)

- Grians, Bernat de (de Grians), (1402, ene. 5-1410, mar. 12) (t.).

- Gomis, Jaume (1397, ago. 4) (t.).

- Despuig, Guerau de (1398, oct. 5) (t.).

- VIDAL, Antoni (Vital) (1406, nov. 29-1407, ene. 14) (t.).

10.5. PORTEROS/PORTARII DOMINI REGIS

- Albaruela, Juan de (1397, nov. 5) (f.).

- GuAsch, Nicolau (1405, mar. 29) (t.).

- Guerau, Francesc (-1410, ene. 16) (t.).

- NANDA, Pere (1405, may. 11) (f.).

- Sanz de Calatayud, Pedro (1403, abr. 23) (f.).

- VeHí, Joan (-1409, mar. 26) (t.).

10.6. ILUMINADOR DE PLOMA DE LA CASA DEL SENYOR REY

- Salvatge, Guillem

10.7. RECEPTOR DE LOS DERECHOS DEL SELLO

- Metge, Bernat. Con Martín I cobró los emolumentos del sello real.

10.8. EXAMINADORES DE NOTARIOS

- Abella (Apilia), Joan de (1404, mar. 5).

- AbelLa, Joan, in legibus licenciatus (1404, jun. 4).

- Almenar (Almanar), Berenguer d' (1408, ene. 31).

- Alós, Gil d' (1400, marz. 26).

- Alsamora (Alçamora) Joan, legum doctor (1402, jul. 5).

- Bardaxí (Bardaxino), Arnaldi de (1398, abr. 17).

- Belluga, Joan, licenciatus in legibus (1402, abr. 8-agos. 22).

- Bosch (de Bosco), Bernat de, in legibus licenciatus (1401, febr. 3-1405, mar. 27).

- Callat, Francesc, legum doctor (1397, dic. 1).

- Camanyes, Blai de (1406, dic. 17).

- Carbonell, Joan (1404, mar. 5).

SCRIPTA, Revista internacional de literatura i cultura medieval i moderna, núm. 6 / desembre 2015 / pp. 1 - 23 
Ma Milagros Cárcel Ortí \& Vicente Pons Alós. La Cancillería de Martín el Humano a través de los Registros Notariorum (1396-1410)

- Cardona, Esperandeu (Sperantem in Deo) (1401, may. 25-1404, febr. 26).

- CARdona, Gabriel (1408, jul. 7).

- CASTElló, Ramon de, jurisperitus (1397, dic. 11-1400, mar. 3).

- «CASTRUeriı», Pere de, legum doctor (1404, nov. 6).

- Català, Pere, licenciatus in decretis (1397, dic. 1).

- CusidA, Joan de (1397, nov. 16).

- Dalmau (Dalmacii), Aymó (1405, ener. 30).

- Descoll (de Colle), Pere, licenciatus in legibus (1397, nov. 20-1409, may. 21).

- Desvall (Vallo), Berenguer, legum doctor (1398, may. 6).

- Ferrer (Ferrarii), Pere (1406, ago. 27).

- Garcia (Garsie), Martí, legum doctor (1408, oct. 17-1410, febr. 25).

- Lanaja, Domingo, jurisperitus (1397, nov. 8).

- LogAYA, Jaume (jurisperitus) (1404, oct. 26).

- Mercader (Mercaderii), Joan (1398, ene. 13-1401, abr. 12).

- Miquel (Michaelis), Bernat, legum doctor (1404, oct. 25).

- Montaner, Pons (1400, febr. 18).

- NArbonés (Narbonesii), Matheus (1398, dic, 17).

- Navarro, Joan, legum doctor (1400, jul. 16-1406, abr. 15).

- Palomar (Palomario), Pere de, legum doctor (1398, mar. 28-may. 15).

- Pére (Petri), Bonanat, legum doctor (1398, febr. 15).

- Pons (Poncii), Andreu (1409, mayo 17).

- Porquet (Porqueti), Bernat (1398, ene. 13-1400, febr. 16).

- «Proponito», Guillem de (1397, dic. 1-1398, jun. 9).

- Sacruelda (Sacruela), Franesc, legum doctor (1409, mar. 26).

- Sant Dionís (Sancto Dionisio), Dalmacius de, legum doctore (1398, jul. 24-1400, nov. 22).

- TORres, Antoni, utriusque iuris professor (1409, ene. 28).

- Urgell (Urgelli), Bernat(1397, dic. 6).

- VAllterra, Joan de (1400, febr. 18).

- Vicent (Vincencii), Joan (1406, jul. 16).

- Vilagayà (Vilagayano/Villagayano), Bernat (1404, nov. 5-1405, jun. 49).

- Ximenez Galloz, Fernando (jurisperitus) (1398, jun. 26).

\section{B. Audiencia}

1. Escribanos De los AUditores/SCRIPTORES AUdITORUM CURIE REGIE/CURIE DICTI DOMINI REGIS/ CURIE DOMINI REGIS/SCRIPTORES AUDIENCIE/

- Ansó, Blasco de (d'Ançano) (1408-1410).

- Besiers, Bartomeu (Beses) (1398, sept. 20-1399, may. 10) (t.).

- Capdevila, Pere de (1400, abr. 8-1403, febr. 13) (t.).

- Destorrent, Pere (1405, oct. 13) (f.).

- Girgós, Tomàs (1398, febr. 6-1400, ene. 27: -1410) (t.)

SCRIPTA, Revista internacional de literatura i cultura medieval i moderna, núm. 6 / desembre 2015 / pp. 1 - 23 
Ma Milagros Cárcel Ortí \& Vicente Pons Alós. La Cancillería de Martín el Humano a través de los Registros Notariorum (1396-1410)

- Besiers, Bartomeu (Veses, Beses), not. (1398, mar. 29-1401, oct. 17; 1410) (t.).

- SARRABlo, Pere (1399, abr. 4) (t.).

2. Verguer/VIRGARIUM AUdIENCIE DOMINI REGIS

- Correger, Arnau (Corregerii) (1408-1410) (t.).

- GARCiA, Joan (1405, ago. 17-1407, ago. 13; 1410) (t.).

- Toyuela (?), Gil de (Coyuela; Toveylla) (1407, ago. 13; 1410) (t.).

\section{ERARio público}

1. TeSORERo (thesAURARIO DOMINI REGIS/DE THESORERLA/DE THESAURARIA DOMINI REGIS)

- Alcanyís, Domingo d' (1404, abr. 14) (f.).

- Bedós, Guillem (Ladors) (1407, jul. 16-sept. 7) (t-f).

- CALles, Guillem (Calleş) (1400, mar. 9) (t.).

- Calonge, Francesc (1400, jul. 10-1408, sept. 27) (t-f).

- Desplà, Joan de, legum doctor (1401, febr. 2-1409, abr. 5) (f.). ${ }^{41}$

- Ferrer, Guillem (1401, ago. 30- 1407, abr. 19) (t-f).

- Guardiola, Andreu (v) (1405, ene. 29) (f.).

- Marí, Pere (1407, abr. 28) (t.).

- Porta, Arnau (1398, jul. 9) (t.).

- Rossell, Joan (1400, jul. 15) (f.).

- RotLÀ, Joan (1402, nov. 3) (f.).

- SACRistà, Joan (1403, ene. 8) (f.).

- SAfont, Antoni de (Anthonius de Fonte) (1409, ene. 20).

- Selma, Jaume (1401, jun. 14) (t.).

- UrgelLÉs, Guillem (v), (1397, jul. 11-1403, may. 12) (t.).

- Verdú, Llorens (1406, abr. 6) (f.).

- IVANYES, Joan (1406, nov. 29) (t.).

2. Mestre RACIONAL/MAGISTER RACIONALIS CURIE DOMINI REGIS

- Artés, Pere d' (1390-1402). ${ }^{42}$

- Fariza, Pedro (1408, sept. 26) (f.).

- Savall (Çavall), Bernat de (1409, nov. 6) (t.).

\section{Capilla del Rey}

1. CAPELLÁN/CAPELLANUS CAPELLE DOMINI REGIS

- Canals, Antoni (1398), maestro en teología, fue lector de la corte real (+1418).

41 Johannes Torregrossa, scutiferius commorans cum Johanne de Plano; Sancius Martini de Tor..., scutiferius de domo domini Johannis de Plano (1401, febr. 2).

42 Está documentado también en la cancillería y tesorería de Juan I (1385-1391).

SCRIPTA, Revista internacional de literatura i cultura medieval i moderna, núm. 6 / desembre 2015 / pp. 1 - 23 
Mª Milagros Cárcel Ortí \& Vicente Pons Alós. La Cancillería de Martín el Humano a través de los Registros Notariorum (1396-1410)

- FERRIS (Ferriç), Joan (1402, ene. 29-1410, abr. 19) (t.), de capella domini regis.

- Gombau, Gabriel (1407), además de capellán aparece como copista de un misal para el rey. - Llobet (Lupera, Lupet), Joan, (1398, ene. 11-1410, abr. 19) (t.)

\section{CONFESOR}

- Baufes, Joan de (1393), obispo de Dax (1375-1391), de Vich (1391-1393) y de Huesca (1393-1403).

- Marí, Pere, O.F.M. (1393, oct. 12).

- Casanova, Joan de, O.P., magister theologie, obispo de Elna (1425-1431) y cardenal de San Sixto (1431-+1436).

\section{E. CASA Y CORTE}

\section{Consejeros Reales}

- Armengol, Joan, doctor en decretos, obispo de Barcelona (...-...) (1398-1408/1399, abr. 27).

- Monçó, Joan de, O.P., doctor en teología por París, universidad de la que fue profesor. Consejero de Martín I desde 1401.

- Malla, Felip de, presbítero de Barcelona. Estudió en Lérida y París y fue su consejero y predicador.

- Metge, Bernat. Con Martín I cobró los emolumentos del sello real y llevaba un cierto control de la cancillería. ${ }^{43}$

\section{CAMARLENGo/CAMARLENGUS DOMINI REGIS)}

- ArTÉs, Pere d' (1390-1402) ${ }^{44}$

- CABrera, Bernat de (1398, marzo 31).

- Centelles, Gilabert de, mossén, camarlengo y conseller del rey (1402, abr. 8).

- Escudero, Sancho (-1409, may. 20) (t.).

- Febrer, Andreu

- Torrelles, Pere de, consiliarius et camarlengus (1408, jul. 5), antes escribano real.

- Sentmenat (Sancto Minato), Galcerà de (1405), miles, consiliarius et camarlengus domini regis

3. CAMARERO/CAMBRER/DE CAMERA DOMINI REGIS

- FERRER, Bernat (1406), membre de la càmara del senyor rey.

- Soler, Francesc de

- Torrelles, Ramon de, domicellus, camerarius (1398, jul. 21).

43 Canellas \& Trenchs, 1988, 74; Mitjà, 1957-1958, 377.

44 Eiximenis le dedicó el Libre del àngels y Antoni Canals la traducción catalana de les Exposicions del Pater Noster, Ave Maria y Salve. Fue embajador de Juan I en París para preparar su boda con Violant de Bar, y en 1407 Martín I le nombró albacea de su testamento (Canellas \& Trenchs, 1988, 74). 
M Milagros Cárcel Ortí \& Vicente Pons Alós. La Cancillería de Martín el Humano a través de los Registros Notariorum (1396-1410)

4. Alguacil/ALgUATZirio

- RajADELl, Guillem de (1397, jul. 30), miles, consiliarius et alguazirius.

5. PANAdero/Pistor DOMINI REGIS

- SALA, Gil de (1407, jun. 23) (f.).

6. Proveedor (EMPTOR DOMINI REGIS)

- Aguilar, Joan (1407, ene. 24) (f.).

7. MÉDICO/APOTHECARIUS DOMINI REGIS

- RicART, Antoni

8. FARMACÉUtico/APOTHECARIUS DOMINI REGIS

- Ledós, Pasqual de, cives Barchinone (1401, oct. 31-1405, mar. 30) (f.).

9. BARBERO/BARBITUNSOR

- Garcia, Blasco (1406, dic. 9) (f.).

10. Peletero (palliparius domus domini regis)

- Granada, Jaume, civis Barchinone (1398, ene. 31- jul. 9) (t-f).

11. ACemilero/ AZEMULarius

- Bierto, Dalmau de (v), domicellus, supraacemularius domini regis (1400, jun. 28) (f.)

12. UJIER DE ARMAS/UXER D'ARMES

- ROCABERTí, Guillem Hug de, uxer d'armes i conceller (1402, abr. 8).

13. ESCUDERO/SCUTIFER DE MUNTERLA DOMINI REGIS)

- LóPEz, Fortún (1401, jun. 20) (f.), scutifer de munteria domini regis.

- Xodes, Eiximen de (1401, jun. 20) (t.).

14. DE DOMO DOMINI REGIS

- Anglesola (Angularia), Pere d' (1402, febr. 13) (f.).

- Ansó, Blasco d' (Danson) (1401, oct. 29) (t.).

- Assió, Joan d' (1402, abr. 15) (t.).

- BAs, Ramon (1398, mar. 21) (t.).

- Bossegays, Jaume de, not. (1399, may. 19) (t.).

- Brau, Martín (1397, dic. 16) (t.).

- Capdevila, Pere de, not. (1399, jun. 18-1402, febr. 3) (t.).

- Català, Pere (-1409, jul. 17) (t.).

- Creixell, Joan, not. (1398, ene. 18-1402, nov. 9) (t.).

- Espigoler, Berenguer, not. (1402, febr. 8- 1405, ene. 20) (t-f).

SCRIPTA, Revista internacional de literatura i cultura medieval i moderna, núm. 6 / desembre 2015 / pp. 1 - 23 
M Milagros Cárcel Ortí \& Vicente Pons Alós. La Cancillería de Martín el Humano a través de los Registros Notariorum (1396-1410)

- Esquerdo, Joan, not. (1398, dic. 12-1399, mar. 14) (t-f).

- Esquerdo, Nicolau (1398, ene. 12) (t.).

- Esteve (Stephani), Joan (1399, abr. 4) (f.).

- Fontelles, Joan de, not. (1402, febr. 3) (t.).

- Geró, Francesc (1405, abr. 29) (f.).

- GIL, Miquel, not. (1398, dic. 12-1402, nov. 9) (t.).

- Girgós, Tomàs (Girgorsii) (1397 sept. 13) (t.).

- GrÀcia, Bartomeu de, not. (1397, sept. 11-1399, febr. 13) (t.).

- GualLart, Martí (1398, mar. 21) (t.).

- Montoliu, Berenguer, not. (1405, may. 29) (f.).

- Péres, Ramon (1397, sept. 11) (t.)

- Pujol, Joan, not. (1407, mar. 22) (f.).

- Roma, Pere, not. (1398, sept. 7-1400, nov. 29) (t.).

- SABATA, Pere (1399, abr. 25) (t.).

- SAFont, Antoni de (Anthonius de Fontes), not. (1406, nov. 24) (f.).

- SAgristà (Sacristani) Joan (1404, ene. 31- febr. 23) (f.).

- SALA, Jaume (1398, ago. 26) (t.).

- Solivella, Francesc de (1399, abr. 25) (t.).

- Subirats, Pere, not. (1402, febr. 8) (t.).

- TARBA, Martín de, not. (1399, may. 19) (t.).

- Tudela, Bertran de (1398, ago. 7-9) (t.).

- Olzinelds, Rafael de (Ulzinellis) (1399, febr. 13) (t.).

- Vilella, Joan (1397, nov. 28) (t.).

- Xipre(?), Benditxo de (1398, febr. 15) (t.).

- ExulBe, (Exulbe)Lluís (1399, abr. 14) (t.).

\section{F. OTros}

1. PRocurator REgIUS IN COMITATIBUS RossiLIONIS ET CERITANIE

- Bierto, Dalmau de, domicellus (v) (1404, mar. 19) (f.).

2. SCRIPTOR OFFICII PROCURACIONIS REGIE COMITATUS ROSSILIONIS ET CERITANIE

- Ferrer, Ramon, not. (1405, dic. 29-1406, febr. 1) (f.).

3. COMMORANS CUM DOMINO HONORABILE PROTHONOTARIO REGIO

- Bossegays, Jacobus de, (1398, ene. 25) (t.).

- Desmas, Pau de (1409, abr. 19) (t.).

- Mataplana, Bernat de (1397, dic. 21-1398, mar. 6) (t.).

- Mura, Antoni de (1410, may. 19) (t.).

- Nicholás, Pau (1402, dic. 21) (t.). 
Ma Milagros Cárcel Ortí \& Vicente Pons Alós. La Cancillería de Martín el Humano a través de los Registros Notariorum (1396-1410)

- ÒDENA, Joan, scriptor (-1408, febr. 14) (t.).

- SALA, Jaume (1398, ene. 25) (t.).

4. COMMORANS CUM HONORABILEM BERENGARIUM SARTA, SECRETARIUM DICTI DOMINI REGIS

- Ferrer, Joan (1404, ago. 1) (t.).

- GARD[...], Garcia de (1404, sept. 10) (t.).

\section{G. Otras casas}

1. Del REy de Sicilia

1.1. SCRIPTORES DOMINI REGIS SICILIE, PRIMOGENITI ARAGONUM (Martí de Sicilia)

- BRuch, Antoni, oriundo del lugar de Alexario, condado de las montañas de Prades, principado de Cataluña, el 3 de abr. de 1405 es nombrado notario per totam terram et dominationem domini regis Aragonum.

- MARín, Ramón, de Ejulve (Aragón), era escribano illustrissimi domini regis Sicilie, es nombrado notario per totam terram et dominacionem el 8 de jul. de 1405.

- Menor, Jaume, escribano de la escribanía del rey de Sicilia (1405, jul. 8) (f.).

- Companyó, Pere (1408, noviembre 8-1409, jul. 15).

2. De la reina Violant de Bar

2.1. Protonotario/PROTHONOTARIUS DOMINE REGINE YOLANDIS

- Besanta, Pere de (1396, noviembre 2-1408, jul. 10) (f.).

- Miquel, Bernat, licenciatus in legibus, consiliaruso et protonotarius (1398, ago. 22-1408, ene. 24)

2.2. ESCRIBANÍA/DE SCRIBANLA DOMINE REGINE

- Gallach, Bernat de (1410, abr. 19)

- Peralta, Pere de, oriundus loci de Peralta, notarius noviter creatus per totam terram et dominacionem domini regis (1405, nov. 12).

- Valls, Antoni (1400, jul. 16-1406, ene. 18).

\subsection{CONFESOR}

- Eiximenis, Joan, O.F.M. (1410)

\subsection{TESORERO/DE THESORERIA DOMINE REGINE}

SANS, Domingo (thesaurarius domine regine Yolandis) (1402, nov. 4) (t.).

2.5. Camareros/CAMERARIUS DOMINE REGINE Yolandis, DOMINI REGIS JoHANNIS, MEMORIE RECOLENDE, RELICTA

- Flasì, Guerau de (1404, may. 30) (t.).

- LluÇà (Luçano), Martí de (1404, may. 30) (f.). 
Ma Milagros Cárcel Ortí \& Vicente Pons Alós. La Cancillería de Martín el Humano a través de los Registros Notariorum (1396-1410)

2.6. DE DOMO DOMINE REGINE

- Pallarés, Galcerà, de domo domine regine Yolandis (1408, oct. 4) (f.).

3. De la Reina Maria

3.1. ESCRIBANÍA/DE SCRIBANIA DOMINE REGINE

- Peralta, Pere de, oriundus loci de Peralta, notarius noviter creatus per totam terram et dominacionem domini regis (1405, nov. 12).

- VAlLs, Antoni (1400, jul. 16-1406, ene. 18).

3.2. ESCRIBANO DE RACIÓN/SCRIPTOR PORCIONIS DOMINE REGINE

- Pujada, Francesc (Pujada, Puyada) (1397, abr. 3-1401, abr. 23) (t-f).

3.3. TESORERO/DE THESORERIA DOMINE REGINE

- Cortilles, Berenguer de (1402, dic. 30) (f.).

3.4. Camarlengo

- Alemany de Cervelló, Lluís, miles, generalis procurator et camarlengus domine regine (1403, may. 2).

3.5. Proveedor (DE OfFici EMPTORIS DOMINE REGINE)

- Comes, Pere (1406, abr. 10) (t.).

3.6. DE DOMO DOMINE REGINE

- Sescomes (Cumbis), Pere de (1403, nov. 22) (t.).

- Prats, Joan (1398, dic. 19) (t.).

- RADA, Mateu de (1401, dic. 20) (t.).

4. De LA INFANTA LEONOR

4.1. DesPENSERO (RABOSTERIUS SERENISSIME DOMINE INFANTE, SORORIS DICTO DOMINI REGIS)

- Serch (Çerch), Ramon de, domicellus (1398, sept. 18) (f.).

5. De la condesa de Foix

5.1. ESCUDERO (SCUTIFERIUS MENSE DE DOMO DOMINE IS ABELIS, COMITISSE FUXENSE)

- Raga, Martín de la (1404, may. 30) (t.).

6. Del obispo de Valencia Hug de Llupià

6.1. SECRETARIO DEL OBISPO

- Pere Llorens (secretarii reverendi domini episcopi Valentini) (1400, may. 11) (f.)

6.2. Proveedor (EMPTor domus epISCopi V ALENTINI)

- Miquel Vidal (oriundus loci dicti castri de Uncastillo, civitatis Dertuse habitator, emptorem domus episcopi Valentini) 
$\mathrm{M}^{\mathrm{a}}$ Milagros Cárcel Ortí \& Vicente Pons Alós. La Cancillería de Martín el Humano a través de los Registros Notariorum (1396-1410)

\section{Abreviaturas}

$\begin{array}{ll}\text { - (f.) } & \text { fideiussor } \\ \text { - not. } & \text { notario } \\ \text { - (s.e.m.) } & \text { sustituto escribano de mandato } \\ \text { - (s.e.r.) } & \text { sustituto escribano de registro } \\ \text { - (t.) } & \text { testigo } \\ \text { - (v.) } & \text { venerable }\end{array}$

SCRIPTA, Revista internacional de literatura i cultura medieval i moderna, núm. 6 / desembre 2015 / pp. 1 - 23 ISSN: 2340-4841 · doi:10.7203/SCRIPTA.6.7008 
Ma Milagros Cárcel Ortí \& Vicente Pons Alós. La Cancillería de Martín el Humano a través de los Registros Notariorum (1396-1410)

\section{Bibliografía}

Aragó Cabañas, A. Ma . (1970) «La escribanía de Juan I», en VIII Congreso de Historia de la Corona de Aragón, II-2, Valencia, pp. 269-293.

Boscolo, A. (1962a) La politica italiana di Martino il Vecchio re d'Aragona, Padova.

- (1962b) «La Sardegna nei primi anni del regno di Martino il Vecchio», Archivio Storico Sardo, 28, pp. 23-41.

Cabanes Catalá, $\mathrm{M}^{a}$. L. (1984) «Fuentes para la historia del notariado. Nombramientos y juramentos de notarios alicantinos», Anales de la Universidad de Alicante. Historia Medieval, III, p. 309-332.

Cabanes Pecourt, $M^{a}$. D. (1993) «Algunos datos sobre nombramientos de notarios generales», en Strenae Emmanuelae Marrero Oblatae, La Laguna, Universidad de Canarias, pp. 225-235.

Canellas, A. \& Trenchs, J. (1988) «Cancillería y cultura. La cultura de los escribanos y notarios de la Corona de Aragón (1344-1479)», Folia Stuttgartensia (Zaragoza, Institución Fernando el Católico), pp. 71-78.

Cárcel Ortí, Må. M. (2008) «Nombramiento de notarios públicos aragoneses (1419-1446)», Aragón en la Edad Media. Homenaje a la Dra. Ma de los Desamparados Cabanes Pecourt, XX, pp. 163-186.

Casula, C. (1973) Il documento regio nella Sardegna aragonese, Padova, CEDAM.

Conde Delgado de Molina, R. \& Gimeno Blay, F. (1986) «Notarías y escribanías de concesión real en la Corona de Aragón (s. XIII)», en Notariado Público y documento privado: de los orígenes al siglo XIV. Actas del VII Congreso Internacional de Diplomática, Valencia, Conselleria de CulturaGeneralitat Valenciana, vol. I, pp. 281-329.

Conde y Delgado de Molina, R. \& Cárcel Ortí, Mª M. (1996) «Corona de Aragón:

Documentación real. Tipología (s. XIII-XIV)», en Diplomatique Royale du Moyen Âge XIII -XIVe Siècles. Actes du Colloque, Porto, Faculdade de Letras, pp. 273-297.

Cruselles Gómez, J. Ma. (1998) Els notaris de la ciutat de València. Activitat professional $i$ comportament social a la primera meitat del segle XV, Barcelona, Fundació Noguera.

D'Arienzo, L. (1974) «Gli scrivani della cancelleria aragonese all'epoca di Pietro il Ceremonioso (1336-1387)», Studi di Paleografía e Diplomatica, Padova, pp. 137-108.

Ferrer i Mallol, $\mathrm{M}^{\mathrm{a}}$. T. (1996) «El Consell reial durant el regnat de Martí l'Humà», en XV Congreso de Historia de la Corona de Aragón, Zaragoza, Departamento de Educación y Cultura, tomo I, vol. 2, pp. 173-190.

. (ed) (2015) Martí l'Humà. El darrer rei de la dinastía de Barcelona (1396-1410). L'interregne i el compromis de Casp, Barcelona, Institut d'Estudis Catalans.

Garrido i Valls, J.D. (2010), Vida i regnat de Martí I: l'ultim rei del Casal de Barcelona, Barcelona, Rafael Dalmau ed. 
Ma Milagros Cárcel Ortí \& Vicente Pons Alós. La Cancillería de Martín el Humano a través de los Registros Notariorum (1396-1410)

Girona Llagostera, D. (1911-1912) «Itinerari del rei en Martí (1396-1402)», Anuari de l'Institut d'Estudis Catalans, IV.

—_. (1913) «Itinerari del rei en Martí (1403-1410)», Anuari de l'Institut d'Estudis Catalans V.

—. (1916) Itinerari del rei en Martí (1396-1402 i 1403-1410), Barcelona, Imp. Henrich.

Graullera Sanz, V. (2000) Los primeros juristas valencianos. Valencia en la Baja Edad Media. Siglos XIII y $X I V$, Valencia, Colegio de Abogados de Valencia.

Günzburg Moll, J. (2001) «La participación de los notarios en las principales instituciones políticas barcelonesas (siglos XIV-XV)», Quaderns d'Història, 5, pp. 47-55.

Mitjà, M. (1957-1958) «Procés contra els consellers, doméstics i curials de Joan I, entre ells Bernat Metge», Boletín de la Real Academia de Buenas Letras, XXVII, pp. 375-417.

Oliva, A. Mª \& Schena, O. (2012) Lettere regie alla città di Cagliari. Le carte reali dell'Archivio comunale di Cagliari. I (1358-1415), Roma, Istituto Storico Italiano.

Pons Alós V. (2005) Cardenales y prelados de Xàtiva en la época de los Borja, Xàtiva, Iglesia Colegial Basílica de Santa María y Centro de Estudios Borgianos.

—. (2012) «Los notarios valencianos en época de Pedro IV y Juan I (1351-1396). Aproximación a su prosopografía», Estudis històrics i documents dels Arxius de Protocols, XXX, pp. 31-86.

- «Me fonc donada la auctoritat de notari. La consolidación de la auctoritas notariae en Valencia en el reinado de Martín el Humano (1396-1410)» (en prensa).

Rodrigo Lizondo, M. (2013) (edició, estudi e indexs a cura de), Col lecció documental de la Cancelleria de la Corona d'Aragó. Textos en llengua catalana (1291-1420). Selecció de textos de Riera i Sans, J., València, Universitat de València, vol. II.

Rodríguez Troncoso, R. (1968) «Documentación notarial del Archivo del Reino de Valencia», en Homenaje a J. Martinez Ferrando, Madrid, pp. 541-556.

Rubió i Balaguer, J. (1961) «Guillem Ponç, secretari del rei Martí, contemporani de Bernat Metge», Estudis Romànics, IX, pp. 67-84.

—_. (1964) «Sobre els orígens de l'humanisme a Catalunya», en Id. La cultura catalana del Renaixement a la Decadència, Barcelona, Publicacions de l'Abadia de Montserrat.

Rubió i Lluch, J. (1908-1921) Documents per l'bistòria de la cultura catalana migeval, Barcelona, Institut d'Estudis Catalans, 2 v. [Edición facsímil Barcelona, IEC, 2000].

Sagarra, F. de (1911). Notes referents als segells del rei Martí, Barcelona, L’Avenç.

Sevillano Colom, F. (1965) «Cancillerías de Fernando I de Antequera y de Alfonso V el Magnánimo», Anuario de Historia del Derecho Español 35, pp. 169-216.

Speranza, V. (2015) Edizione e studio di fonti per la storia della Puglia nel periodo di Alfonso il Magnanimo, Barcelona, Universitat de Barcelona. Tesis de Doctorado inédita. 
Ma Milagros Cárcel Ortí \& Vicente Pons Alós. La Cancillería de Martín el Humano a través de los Registros Notariorum (1396-1410)

Torra Pérez, A. (1994) «Los registros notariorum de la Cancillería Real Aragonesa», Napoli Nobilissima. Rivista di Arti figurativi, archeologia e urbanistica, XXXIII, maggio-dicembre, pp. 179-194.

Trenchs Odena, J. (2011) Documents de Cancelleria $i$ de Mestre Racional sobre la cultura catalana medieval (Ferrer i Mallol, Ma T. coord.), Barcelona, IEC.

Trenchs, J. \& Aragó, A.Ma . (1983) «Las cancillerías de la Corona de Aragón y Mallorca desde Jaime I a la muerte de Juan II», Folia Parisiensia, 1, Zaragoza, Institución Fernando el Católico-CSIC.

Udina Martorell, F. (1986) Guía histórica y descriptiva del Archivo de la Corona de Aragón, Madrid, Dirección General de Bellas Artes y Archivos. 